\title{
Identification of divacancy and silicon vacancy qubits in $6 \mathrm{H}-\mathrm{SiC}$
}

J oel Davidsson, Viktor Ivády, Rickard Armiento, Takeshi Ohshima, Son Tien Nguyen, Adam Gali and Igor Abrikosov

The self-archived postprint version of this journal article is available at Linköping University Institutional Repository (DiVA):

http:// urn.kb.se/ resolve?urn=urn:nbn:se:liu:diva- 156202

N.B.: When citing this work, cite the original publication.

Davidsson, J., Ivády, V., Armiento, R., Ohshima, T., Nguyen, S. T., Gali, A., Abrikosov, I., (2019),

Identification of divacancy and silicon vacancy qubits in 6H-SiC, Applied Physics Letters, 114(11), 112107. https:/ / doi.org/ 10.1063/ 1.5083031

Original publication available at:

https:// doi.org/ 10.1063/ 1.5083031

Copyright: AIP Publishing

http:// www.aip.org// 


\title{
Identification of divacancy and silicon vacancy qubits in $6 \mathrm{H}-\mathrm{SiC}$
}

\author{
Joel Davidsson, ${ }^{1,}$ a) Viktor Ivády, ${ }^{1,2}$ Rickard Armiento, ${ }^{1}$ Takeshi Ohshima, ${ }^{3}$ N.T. Son, ${ }^{1}$ Adam Gali, ${ }^{2,4}$ and \\ Igor A. Abrikosov ${ }^{1,5}$ \\ ${ }^{1)}$ Department of Physics, Chemistry and Biology, Linköping University, SE-581 83 Linköping, \\ Sweden \\ ${ }^{2)}$ Wigner Research Centre for Physics, Hungarian Academy of Sciences, PO Box 49, H-1525, Budapest, \\ Hungary \\ ${ }^{3)}$ National Institutes for Quantum and Radiological Science and Technology, 1233 Watanuki, Takasaki, Gunma 370-1292, \\ Japan \\ ${ }^{4)}$ Department of Atomic Physics, Budapest University of Technology and Economics, Budafoki út 8., H-1111 Budapest, \\ Hungary \\ ${ }^{5)}$ Materials Modeling and Development Laboratory, National University of Science and Technology 'MISIS', 119049 Moscow, \\ Russia
}

Point defects in semiconductors are relevant for use in quantum technologies as room temperature qubits and single photon emitters. Among suggested defects for these applications are the negatively charged silicon vacancy and the neutral divacancy in $\mathrm{SiC}$. The possible nonequivalent configurations of these defects have been identified in $4 \mathrm{H}-\mathrm{SiC}$, but for $6 \mathrm{H}-\mathrm{SiC}$ the work is still in progress. In this paper, we identify the different configurations of the silicon vacancy and the divacancy defects to each of the V1-V3 and the QL1-QL6 color centers in 6H-SiC, respectively. We accomplish this by comparing results from ab initio calculations with experimental measurements for zero-phonon line, hyperfine tensor, and zero-field splitting.

In recent years, point defects in semiconductors have been suggested for implementing quantum bits (qubit) ${ }^{1-3}$ and single photon sources ${ }^{4-8}$ for quantum computation, ${ }^{9}$ quantum information processing,,$^{10}$ spintronics, ${ }^{3}$ and quantum sensing applications. ${ }^{11-13}$ The most studied point defect qubits are the negatively charged nitrogen-vacancy center (NV center) in diamond, ${ }^{1,14}$ the neutral divacancy in $\mathrm{SiC},{ }^{15,16}$ and the negatively charged silicon vacancy in SiC. ${ }^{17,18}$ All of these defects exhibit well isolated electron spin states with long coherence time and operate even at room temperature. ${ }^{18-20} \mathrm{SiC}$ is a technologically mature host for qubits and single photon emitters, which makes it possible to integrate quantum technologies and semiconductor devices. There are numerous polytypes of $\mathrm{SiC}$ that often host multiple symmetrically nonequivalent $\mathrm{Si}$ and $\mathrm{C}$ sites in their primitive cell. Consequently, point defect qubits may have several nonequivalent configurations with different characteristics in each polytype that provide an alternative tools for engineering qubit properties in SiC. ${ }^{16}$ Assigning the experimental photoluminescence (PL) and electron spin resonance (ESR) signals to the nonequivalent configurations is indispensable for deeper understanding of the qubits. Recently, several works have been published on the identification of the microscopic structure of point defect qubits in $4 \mathrm{H}-\mathrm{SiC} .^{21-23}$

The $4 \mathrm{H}$ and $6 \mathrm{H}$ polytypes are the most commonly used hexagonal polytypes of $\mathrm{SiC}$ with wafer size samples and high quality. For $6 \mathrm{H}-\mathrm{SiC}$, there are 12 atoms in the primitive cell with 3 non-equivalent sites for both species, see Fig. 1(a). Considering only the immediate vicinity of the sites, one site has hexagonal like environment, while the other two sites have cubic like environments. These sites are commonly labeled as $h, k 1$ and $k 2$ (see Fig. 1(a)), respectively. In general, 6H$\mathrm{SiC}$ gives rise to 3 configurations for single site point defects,

\footnotetext{
${ }^{a)}$ Electronic mail: joel.davidsson@liu.se
}

such as the silicon vacancy, and 6 configurations for pair defects, such as the divacancy. For the silicon vacancy, the configurations are named $h, k 1$, and $k 2$, all of which have $C_{3 v}$ point group symmetry. The related photoluminescence (PL) signals are referred to as $\mathrm{V} 1-\mathrm{V} 3^{24}$ centers. Optically detected magnetic resonance (ODMR) and electron paramagnetic resonance (EPR) centers are referred to as $\mathrm{T}_{\mathrm{V} 1 \mathrm{a}}-\mathrm{T}_{\mathrm{V} 3 \mathrm{a}}$ centers ${ }^{24}$ and $\mathrm{V}_{\mathrm{Si}}^{-}(\mathrm{I})-\mathrm{V}_{\mathrm{Si}}^{-}(\mathrm{II})$ centers. ${ }^{25}$ For the divacancy, we use the notation $\mathrm{V}_{\mathrm{Si}^{-}}-\mathrm{V}_{\mathrm{C}}$, thus the following configurations are possible; $h h, k 1 k 1$, and $k 2 k 2$ with $C_{3 v}$ point group symmetry, and $h k 2, k 1 h$, and $k 2 k 1$ with $C_{1 h}$ point group symmetry. Divacancy defects in $6 \mathrm{H}-\mathrm{SiC}$ give rise to the QL1-QL6 ${ }^{16} \mathrm{PL}$ and ESR centers P6-P7 ${ }^{26}$ respectively. The symmetry arguments for the divacnancy and silicon vacancy in $6 \mathrm{H}-\mathrm{SiC}$ are the same as in $4 \mathrm{H}-\mathrm{SiC} .27,28$
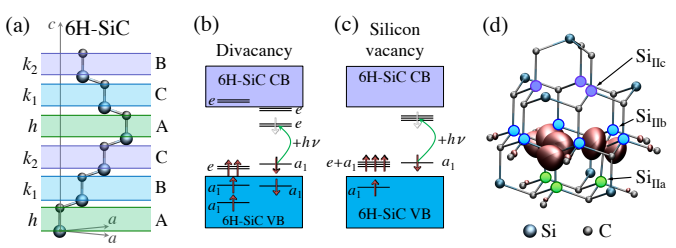

FIG. 1: (color online) (a) depicts the primitive cell of $6 \mathrm{H}-\mathrm{SiC}$. Light green, light blue, and light purple bands highlight the hexagonal-like $(h)$ and the two different cubic-like ( $k 1$ and $k 2$ ) Si-C double layers. (b) and (c) depict a schematic diagram of the Kohn-Sham electronic structure of the neutral divacancy and negatively charged silicon vacancy, respectively. CB stands for conduction band and

VB for valance band. Green arrows represent optical absorption processes that drive the defects into their lowest energy optically excited state. (d) Configuration and spin density of the $h h$ divacancy. ${ }^{29} \mathrm{Si}$ sites considered in the hyperfine tensor calculations are marked with colored circles.

The theoretical description and engineering of the defect 
centers require the assignment of each of the different microscopic configurations. This has been done for the silicon vacancy $^{22}$ and the divacancy in $4 \mathrm{H}-\mathrm{SiC}^{21,23,27}$ but for $6 \mathrm{H}-\mathrm{SiC}$ the work is still in progress. In this paper, we present an accurate identification of the different configurations for the divacancy and silicon vacancy point defects in $6 \mathrm{H}-\mathrm{SiC}$. To assign the different configurations, we use the approach of comparing experimental measurements with first-principle theoretical calculations. The properties used for this comparison are the zero-phonon line (ZPL) energy, the zero-field splitting (ZFS) parameter, and hyperfine splitting due to the first and second neighbor nuclear spins.

To calculate the ZPL, ZFS, and hyperfine parameters we employ density functional theory ${ }^{29-31}$ (DFT). The calculations are performed by using the Vienna $\mathrm{Ab}$ initio Simulation Package (VASP), ${ }^{32,33}$ which uses projector augmented wave (PAW) method ${ }^{34,35}$ for core electrons and plane wave basis set for valence electrons. For exchange-correlation, we use the semi-local functional of Perdew, Ernzerhof, and Burke $(\mathrm{PBE})^{36}$ and the non-local range-separated hybrid functional of Heyd, Scuseria, and Ernzerhof (HSE06). ${ }^{37,38}$

The calculations with the PBE functional are carried out with a plane wave energy and kinetic energy cutoff of $420 \mathrm{eV}$ and $840 \mathrm{eV}$ respectively. The energy criterion for the selfconsistent cycle and the structural relaxation are set to $10^{-5}$ $\mathrm{eV}$ and $10^{-3} \mathrm{eV}$, respectively. For the HSE functional calculations, the plane wave energy and kinetic energy cutoff are the same as for the PBE functional. The energy convergence criterion is lowered to $10^{-4} \mathrm{eV}$. For the hybrid functional computations, the grid for the Fast Fourier Transformation (FFT) for the semi-local exchange is set to twice the largest wave vector, for the exact exchange it is set to the largest wave vector in order to reduce wrap-around errors and produce good energies respectively.

For the ZPL energy calculation, we use the constrained occupation DFT method. ${ }^{39}$ The lowest energy optically excited state is calculated by promoting a Kohn-Sham particle from the highest occupied state to the lowest unoccupied state in the minority spin channel, see Fig. 1(b)-(c). PBE can find the correct order for the ZPL energies of the non-equivalent configurations, but the absolute values are shifted down by $0.2-0.3 \mathrm{eV}$ depending on supercell size. ${ }^{21}$ The ground state PBE wave functions are used to calculate the zero-field splitting (ZFS) tensor employing the implementation in VASP as well as the method presented in Ref. 40. The latter method is called inhouse throughtout this paper. Both implementations calculate the spin-spin dipole interaction which is the first order approximation of the ZFS. Note that the latter implementation ignores the PAW contribution to the ZFS, it produces good absolute values while the implementation in VASP is formally more consistent.

The results of the HSE06 ground state calculations are postprocessed to obtain the hyperfine field tensor ${ }^{41}$ using the implementation present in VASP. This tensor describes the small energy splitting due to the interaction between the nuclear and electronic spin. Due to the higher natural abundance of the spin- $1 / 2{ }^{29} \mathrm{Si}$ isotope (4.68\%) than the spin- $1 / 2{ }^{13} \mathrm{C}$ isotope $(1.07 \%)$, it is easier to resolve the hyperfine signal of ${ }^{29} \mathrm{Si}$ in experiment. We calculate the hyperfine tensor of the second neighbor ${ }^{29} \mathrm{Si}$ nuclei sites for the divacancy. The different ${ }^{29} \mathrm{Si}$ nuclei sites are displayed in Fig. 1(d). For the silicon vacancy, the hyperfine tensors of ${ }^{13} \mathrm{C}$ for the silicon vacancy are calculated for the carbon atoms directly above the defect $\left(\mathrm{C}_{1}\right)$ and for the three carbon atoms below $\left(\mathrm{C}_{2}-\mathrm{C}_{4}\right)$.

We also perform EPR experiments for the silicon vacancy in $6 \mathrm{H}-\mathrm{SiC}$ at room temperture. The samples used for EPR experiments are high-purity semi-insulating $6 \mathrm{H}-\mathrm{SiC}$ irradiated by $2-\mathrm{MeV}$ electrons at room temperature followed by a annealing at $400^{\circ} \mathrm{C}$. The dose of irradiation was $8 \times 10^{18} \mathrm{~cm}^{-2}$. EPR measurements are performed on an X-band $(9.4 \mathrm{GHz}$ ) spectrometer equipped with a He-flow cryostat, allowing the regulation of the sample temperature in the range 4-295 K.

In this paper, a 1536 atom supercell $(8 \times 8 \times 2)$, with basis vector length of $24.8 \AA, 24.8 \AA$, and $30.4 \AA$ is used. The supercell size together with $\Gamma$ point sampling is sufficiently convergent for point defect configuration identification. ${ }^{21,22}$ For the relative energy difference of the ZPL energy, we fitted a normal distribution with a standard deviation of $4.26 \mathrm{meV}$, which would correspond to a Full Width at Half Maximum (FWHM) of $10 \mathrm{meV}$. Here, we assume that $10 \mathrm{meV}$ away from the calculated value, the probability of finding the correct ZPL drops to half. As it was discussed in Ref. 21 and evident from Fig. 2, a complete identification cannot be obtained from the ZPL results alone. (a) $\mathrm{V}_{\mathrm{Si}}(-)$

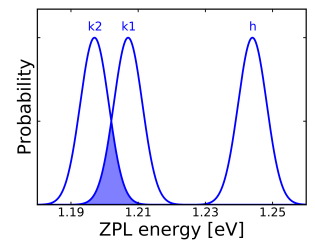

(b) $\mathrm{V}_{\mathrm{Si}}-\mathrm{V}_{\mathrm{C}}$

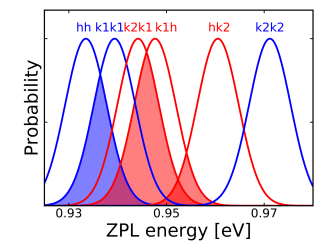

FIG. 2: Theoretical ZPL lines in 6H-SiC from DFT computations using the PBE functional. Estimated errors are depicted by Gaussians with a standard deviation of $4.2 \mathrm{meV}$. Shown are (a) the negatively charged silicon vacancy, and (b) the neutral divacancy with the overlap filled in. Blue (red) color is for configurations with $C_{3 v}\left(C_{1 h}\right)$ symmetry.

Fig. 2(a) shows the calculated ZPL energies for the silicon vacancy. As can be seen in the figure, the theoretical ZPL lines for the $k 1$ and $k 2$ overlap slightly, hence it is possible that these lines have a different order when compared to the experiments. The ZPL for the $h$ site, however, has no overlap with other sites, thus the V1 center of the largest ZPL energy can be assigned to $\mathrm{V}_{\mathrm{Si}}(-)$ at the $h$ site.

In Fig. 2(b) for the divacancy ZPLs, the largest overlap is between $k 1 h$ and $k 2 k 1$ also $h h$ and $k 1 k 1$, therefore no assignment can be made directly. In contrast, since the ZPL lines for $k 2 k 2$ do not overlap with any other $\mathrm{C}_{3 \mathrm{v}}$ symmetric configurations, this configuration can be assigned to the highest energy divacancy related center, QL6. Note, also, that the order of $k 1 k 1$ and $k 2 k 1$ is clear when comparing to the experimental data, since they belong to different symmetry groups.

As divacancy defects have more applications, their results are discussed first. If one only use the PBE ZPL data pre- 
TABLE I: Theoretical and experimental magneto-optical data for divacancy configurations in $6 \mathrm{H}-\mathrm{SiC}$.

\begin{tabular}{|c|c|c|c|c|c|}
\hline \multirow{2}{*}{$\begin{array}{l}\text { Configuration } \\
\mathrm{V}_{\mathrm{Si}}-\mathrm{V}_{\mathrm{C}}\end{array}$} & \multirow{2}{*}{ Center $^{15,16}$} & \multirow{2}{*}{$\begin{array}{c}\text { ZPL }[\mathrm{eV}] \\
\text { Calc. Exp. }{ }^{16}\end{array}$} & \multicolumn{3}{|c|}{ ZFS [GHz] } \\
\hline & & & $\begin{array}{l}\text { Calc. } \\
\text { (VASP) }\end{array}$ & $\begin{array}{c}\text { Calc. } \\
\text { (inhouse) }\end{array}$ & Exp. ${ }^{16}$ \\
\hline$\overline{k 1 k 1}$ & QL1 & $0.939 \quad 1.088$ & 1.494 & 1.300 & 1.300 \\
\hline$h h$ & QL2 & $\begin{array}{ll}0.933 & 1.092\end{array}$ & 1.552 & 1.342 & 1.334 \\
\hline$k 1 h$ & QL3 & $0.948 \quad 1.103$ & 1.521 & 1.335 & 1.236 \\
\hline$k 2 k 1$ & QL4 & $0.944 \quad 1.119$ & 1.549 & 1.343 & - \\
\hline$h k 2$ & QL5 & $0.961 \quad 1.134$ & 1.559 & 1.355 & 1.383 \\
\hline$k 2 k 2$ & QL6 & $0.972 \quad 1.134$ & 1.579 & 1.374 & 1.347 \\
\hline
\end{tabular}

TABLE II: Theoretical and experimental hyperfine splitting for divacancy configurations in $6 \mathrm{H}-\mathrm{SiC}$ for the $C_{3 v}$ symmetry configurations. The sites of the nuclei considered in the hyperfine calculations are marked in Fig. 1(d). The numbers in parentheses show the multiplicity of the sites.

The hyperfine splitting $[\mathrm{MHz}]$ is $A_{z}$ with correction.

\begin{tabular}{|c|c|c|c|c|c|}
\hline \multirow{2}{*}{$\begin{array}{l}\text { Configuration } \\
V_{S_{i}}-V_{C}\end{array}$} & \multirow{2}{*}{ Center $^{15,16}$} & \multicolumn{2}{|c|}{$\mathrm{Si}_{\mathrm{IIa}}(3)$} & \multicolumn{2}{|c|}{$\operatorname{Si}_{I I b}(6)$} \\
\hline & & Calc. & Exp. $^{42}$ & Calc. & Exp. $^{42}$ \\
\hline$k 1 k 1$ & QL1 & 12.70 & 12.7 & 9.46 & 10.0 \\
\hline$h h$ & QL2 & 11.72 & 12.5 & 9.10 & 9.2 \\
\hline$k 2 k 2$ & QL6 & 11.72 & 13.3 & 9.23 & 9.2 \\
\hline
\end{tabular}

sented in Table I for the divacancy, it suggests the following identification for QL1-QL6 in increasing order: $h h, k 1 k 1$, $k 2 k 1, k 1 h, h k 2$, and $k 2 k 2$. However, when the ZFS is also taken into account, one finds that a better agreement is given by reordering the identified configurations so that $h h$ switch with $k 1 k 1$ and $k 2 k 1$ switch with $k 1 h$. As discussed above $h h$ and $k 1 k 1$ as well as $k 2 k 1$ and $k 1 h$ have a large overlap in the ZPL data and this reordering gives better agreement with all the available data. Furthermore, taking the hyperfine splitting data in Table II into account, further strengthens the decision to switch $h h$ with $k 1 k 1$. The final results of our identification are given in Table I.

In our identification, the $C_{3 v}$ configurations ( $h h, k 1 k 1$, and $k 2 k 2$ ) are the most accurate given that all the data (ZPL, ZFS, and hyperfine) support this order. For the $C_{1 h}$ configurations ( $h k 2, k 1 h$, and $k 2 k 1$ ), the results are consistent with the data presented here, but further hyperfine measurements are needed to verify the presented assignment of the configurations. In the supplementary material, additional hyperfine tensors are calculated to facilitate the interpretation of future experimental results. Additional experimental results would be especially useful for the $k 1 h$ and $k 2 k 1$ configurations where ZPL values have a large overlap and the ZFS value is missing for the $k 2 k 1$ configuration. On the other hand, the $h k 2$, which does not have a large overlap with $k 1 h$ and $k 2 k 1$ in Fig. 2(b), is consistent with both the ZFS and ZPL data. Note, also, that in the $\mathrm{C}_{1 h}$ configurations, spin-orbit interaction may contribute to the ZFS. As we only calculate the spin-spin dipolar contribution, we expect larger errors when the calculated ZFS is compared with the experimental values in these cases.

If one uses only the PBE ZPL data for the silicon vacancy, one would get an identification of V1-V3 centers in increasing order: $k 2, k 1$, and $h$. However, in similarity with the case for
TABLE III: Theoretical and experimental magneto-optical data for silicon vacancy configurations in $6 \mathrm{H}-\mathrm{SiC}$.

\begin{tabular}{l|l||rr|rrr}
\hline \hline \multirow{2}{*}{ Configuration } & \multirow{2}{*}{ Center $^{24}$} & \multicolumn{2}{|c|}{ ZPL [eV] } & \multicolumn{3}{|c}{ ZFS [MHz] } \\
& & Calc. Exp. & Calc. & Calc. & Exp. \\
& & & & VASP) & (inhouse) & $D_{\text {exp }} \mid$ \\
\hline$k 1$ & V3, T $_{\text {V3a }}$ & 1.207 & 1.368 & -32.8 & -26.7 & 13.9 \\
$k 2$ & V2, T $_{\text {V2a }}$ & 1.197 & 1.398 & 97.5 & 97 & 64.0 \\
$h$ & V1, T & 1.244 & 1.434 & 9.7 & 3.7 & 13.3 \\
\hline \hline
\end{tabular}

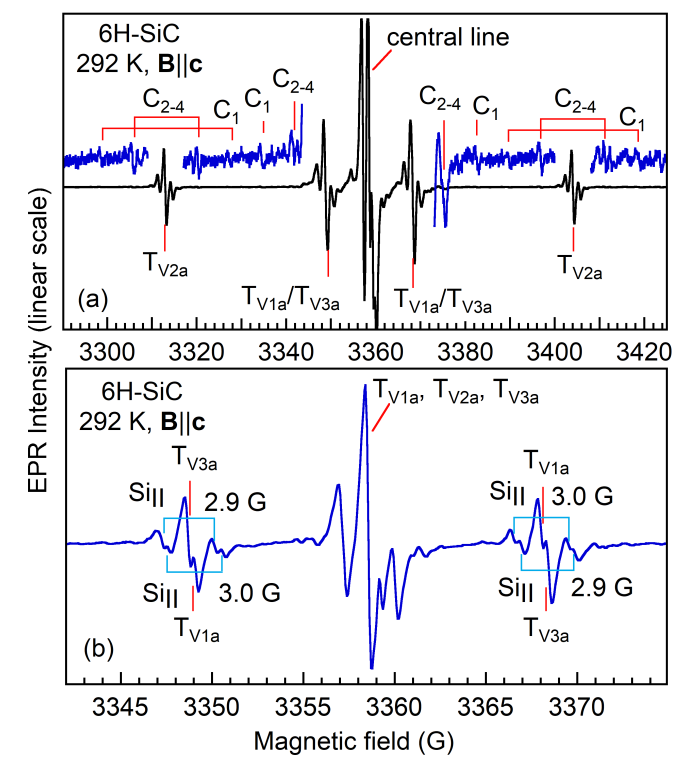

FIG. 3: (a) EPR spectra in electron-irradiated high-purity semi-insulating $6 \mathrm{H}-\mathrm{SiC}$ measured at $292 \mathrm{~K}$ for $\mathbf{B} \| \mathbf{c}$ showing

the low- and high-field lines and the central line of the negative $S i$ vacancies $T_{\mathrm{v} 1 \mathrm{a}}, \mathrm{T}_{\mathrm{V} 2 \mathrm{a}}$, and $\mathrm{T}_{\mathrm{V} 3 \mathrm{a}}$ as well as the $\mathrm{C}_{1}$ and $\mathrm{C}_{2-4}$ hyperfine structures. With the modulation field of $0.7 \mathrm{G}$ and the $\mathrm{MW}$ power of $1.262 \mathrm{~mW}$, the signals of $\mathrm{T}_{\mathrm{V} 1 \mathrm{a}}$ and $\mathrm{T}_{\mathrm{V} 3 \mathrm{a}}$ are not resolved. (b) Using a modulation field of

$0.6 \mathrm{G}$ and and $\mathrm{MW}$ power of $0.6325 \mathrm{~mW}$, the low- and

high-field lines of $\mathrm{T}_{\mathrm{V1a}}$ and $\mathrm{T}_{\mathrm{V} 3 \mathrm{a}}$ as well as their $\mathrm{Si}_{\mathrm{II}}$ hyperfine structures could be resolved. The MW frequency is $9.415 \mathrm{GHz}$.

the divacancy, when one takes the ZFS results into account, the order of 2 adjacent configurations change. In this case, the order changes between $k 1$ and $k 2$, as seen in Table III. Since the calculated ZFS is the smallest for the $h$ site, $9.7 \mathrm{MHz}$ from VASP and 3.7 MHz from the inhouse method, it matches well with the smallest experimental value of $13.3 \mathrm{MHz}$. The calculated largest ZFS value belongs to $k 2,97.5 \mathrm{MHz}$ and $97 \mathrm{MHz}$ for the two computational techniques respectively, which is matched with the largest experimental value $64.0 \mathrm{MHz}$. The ZFS of the $k 1$ configuration does not fit the experimental value and thus does not aid the identification. Our EPR setup only measures the amplitude of the D-tensor. The ZFS results for the $k 1$ and $k 2$ configurations agree with the calculation and experiment done in Ref. 44, where the different signs of these configurations are explained.

Fig. 3(a) shows an EPR spectrum in irradiated 6H-SiC measured at $292 \mathrm{~K}$ for the magnetic field along the c-axis $(\mathbf{B} \| \mathbf{c})$. Here, the hyperfine structures due to the interaction between 
TABLE IV: Theoretical and experimental hyperfine splitting [MHz] for silicon vacancy configurations in $6 \mathrm{H}-\mathrm{SiC}$ for the nuclei site (a) $\mathrm{C}_{1}$ and sites (b) $\mathrm{C}_{2-4}$ are presented with the same labeling as in Ref. 25.

\begin{tabular}{|c|c|c|c|c|c|c|c|}
\hline \multirow{2}{*}{ Configuration } & \multirow{2}{*}{ Center $^{24,25}$} & \multicolumn{2}{|c|}{ Calc. } & \multicolumn{2}{|c|}{ Exp. } & \multicolumn{2}{|c|}{ Exp. $^{25}$} \\
\hline & & $\overline{\mathrm{A}_{\|}}$ & $\mathrm{A}_{\perp}$ & $\mathrm{A}_{\|}$ & $\mathrm{A}_{\perp}$ & $\overline{\mathrm{A}_{\|}}$ & $\overline{\mathrm{A}_{\perp}}$ \\
\hline$k 1$ & $\mathrm{~T}_{\mathrm{V} 3 \mathrm{a}}, \mathrm{V}_{\mathrm{Si}}^{-}(\mathrm{I})$ & 84.3 & 30.3 & 80.5 & 33.6 & 80.0 & 32.8 \\
\hline$k 2$ & $\mathrm{~T}_{\mathrm{V} 2 \mathrm{a}}$ & 83.3 & 33.2 & 80.5 & 33.0 & - & - \\
\hline$h$ & $\mathrm{~T}_{\mathrm{V} 1 \mathrm{a}}, \mathrm{V}_{\mathrm{Si}}^{-}(\mathrm{II})$ & 84.6 & 31.2 & 80.5 & 33.6 & 80.0 & 32.8 \\
\hline
\end{tabular}

(a) The parallel and perpendicular components are presented from our calculation, measurements, and from Ref. 25.

\begin{tabular}{|c|c|c|c|c|c|c|c|}
\hline \multirow{2}{*}{ Configuration } & \multirow{2}{*}{ Center $^{24,25}$} & \multicolumn{3}{|c|}{ Calc. } & \multicolumn{3}{|c|}{ Exp. $^{25}$} \\
\hline & & $\mathrm{A}_{\mathrm{xx}}(\theta)$ & $\mathrm{A}_{\mathrm{yy}}(90)$ & $\mathrm{A}_{\mathrm{zz}}(\theta)$ & $\mathrm{A}_{\mathrm{xx}}(\theta)$ & $\mathrm{A}_{\mathrm{yy}}(90)$ & $\mathrm{A}_{\mathrm{zz}}(\theta)$ \\
\hline$k 1$ & $\mathrm{~T}_{\mathrm{V} 3 \mathrm{a}}, \mathrm{V}_{\mathrm{Si}}^{-}(\mathrm{I})$ & $79.9(109.7)$ & 26.6 & $27.0(19.7)$ & $75.8(110.0)$ & 28.4 & $\overline{28.2(20.0)}$ \\
\hline$k 2$ & $\mathrm{~T}_{\mathrm{V} 2 \mathrm{a}}$ & 85.3(109.6) & 30.8 & $30.8(19.6)$ & - & - & - \\
\hline$h$ & $\mathrm{~T}_{\mathrm{V} 1 \mathrm{a}}, \mathrm{V}_{\mathrm{Si}}^{-}(\mathrm{II})$ & 83.6(108.9) & 30.1 & $30.1(18.9)$ & $80.3(109.1)$ & 31.6 & $31.4(19.1)$ \\
\hline
\end{tabular}

(b) For the three degenerate $\mathrm{C}_{2-4}$ sites, the $\mathrm{A}_{\mathrm{xx}}, \mathrm{A}_{\mathrm{yy}}$, and $\mathrm{A}_{\mathrm{zz}}$ components with their corresponding $\theta$ value are presented and compared.

the electron spin and the nuclear spin of one ${ }^{13} \mathrm{C}$ occupying one of the four nearest neighbor $\mathrm{C}_{1}$ (along the c-axis) and $\mathrm{C}_{2-4}$ (in basal plane) sites could be detected. However, the $\mathrm{T}_{\mathrm{V} 1 \mathrm{a}}$ and $\mathrm{T}_{\mathrm{V} 3 \mathrm{a}}$ signals could not be resolved, resulting in overlapping lines with a double intensity (see Fig. 3(a)). Using smaller field modulation $(0.6 \mathrm{G})$ and microwave (MW) power $(0.6325 \mathrm{~mW})$, the $\mathrm{T}_{\mathrm{V} 1 \mathrm{a}}$ and $\mathrm{T}_{\mathrm{V} 3 \mathrm{a}}$ lines and their hyperfine structures due to the interaction with one ${ }^{29} \mathrm{Si}$ occupying one of the $12 \mathrm{Si}$ sites in the second neighbor can be observed, see Fig. 3(b). The fine-structure parameters D for these $S=3 / 2$ centers are determined as: $13.3 \mathrm{MHz}$ for $\mathrm{T}_{\mathrm{V} 1 \mathrm{a}}, 64.0 \mathrm{MHz}$ for $\mathrm{T}_{\mathrm{V} 2 \mathrm{a}}$ and $13.9 \mathrm{MHz}$ for $\mathrm{T}_{\mathrm{V} 3 \mathrm{a}}$, see Table III. The isotropic $\mathrm{Si}_{\text {II }}$ hyperfine splittings for these centers are determined as: 8.4 MHz for $\mathrm{T}_{\mathrm{V} 1 \mathrm{a}}$ and $8.1 \mathrm{MHz}$ for $\mathrm{T}_{\mathrm{V} 2 \mathrm{a}}$ and $\mathrm{T}_{\mathrm{V} 3 \mathrm{a}}$. Note that the fine-structure parameter for the $h$ configuration reported in Ref. 44 is $D=0$ whereas in our experiments $D=13.3 \mathrm{MHz}$. In Ref. 44, linewidth of electron spin echo (ESE) is very broad ( $5 \mathrm{G}$ full width at half maximum) and the $\mathrm{V}_{\mathrm{Si}}^{-}(h)$ line (i.e. $\mathrm{T}_{\mathrm{V} 1 \mathrm{a}}$ ) and $\mathrm{V}_{\mathrm{Si}}^{-}(k 1)$ line (i.e. $\mathrm{T}_{\mathrm{V} 3 \mathrm{a}}$ ) cannot be resolved. The two observed doublets were assigned to $k 1$ and $k 2$ configurations whereas $h$ was assumed to have no ZFS with its lowand high- field lines coincide with the central line. ${ }^{44}$

In Table IV, we compare the calculated principal values for the hyperfine tensors and corresponding $\theta$ angles with the experimental data. ${ }^{25}$ This tensor has values labeled $A_{x x}$,

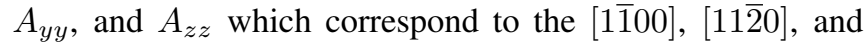
[0001]-direction and $\theta$ is the angle to the [0001]-direction (cf. Fig. 1(c) in Ref. 25, where the same values are denoted $A_{x}$, $A_{y}$, and $A_{z}$ ). For nuclei in high symmetric positions, such as $\mathrm{C}_{1}, A_{x x}=A_{y y}$ and thus denoted $A_{\perp}$ with a $\theta=90$ whereas the $A_{z z}$ is denoted $A_{\|}$with a $\theta=0$. These hyperfine data of the nearest neighboring $\mathrm{C}_{1}$ and $\mathrm{C}_{2-4}$ of the $\mathrm{Si}$ vacancy were measured from the central line, which were considered to be related to the so-called no-ZFS negative $\mathrm{Si}$ vacancies $\mathrm{V}_{\mathrm{Si}}(\mathrm{I})$ and $\mathrm{V}_{\mathrm{Si}}(\mathrm{II}) .{ }^{25}$ The corresponding $\mathrm{C}_{1}$ hyperfine data for the $\mathrm{T}_{\mathrm{V} 1 \mathrm{a}}, \mathrm{T}_{\mathrm{V} 2 \mathrm{a}}$, and $\mathrm{T}_{\mathrm{V} 3 \mathrm{a}}$ determined from our EPR experiments are also given. The $\mathrm{C}_{1}$ and $\mathrm{C}_{2-4}$ hyperfine structures for the low- and high field lines of these centers are shown in Fig. 3(a). In Table IVa, the calculated $A_{\perp}$ and $A_{\|}$for the $\mathrm{C}_{1}$ nuclei for $k 1$ and $h$ are very close to experiment and thus correspond to $\mathrm{T}_{\mathrm{V} 1 \mathrm{a}}$ and $\mathrm{T}_{\mathrm{V} 3 \mathrm{a}}$. This conclusion is further strengthen by the 2 equal values reported in Ref. 25. In Ta- ble IVb, we compare the calculated results for $A_{x x}, A_{y y}, A_{z z}$, and the corresponding $\theta$ angle with the experimental data ${ }^{25}$ for the $\mathrm{C}_{2-4}$ sites. Here, the presented experimental data also agrees best with the $k 1$ and $h$ configurations. Due to the small angle difference between $k 1$ and $k 2$, it could be difficult to resolve the different amplitudes for these configurations.

When comparing the errors between the theoretical and experimental ZPL values in Table I and Table III, there is a fairly uniform systematic positive offset with an average mangintude of $0.15 \mathrm{eV}$ for these configurations. The systematic error originates from the use of the PBE functional, and has been thoroughly discussed in Ref. 21. Also, note that the ZFS values for the divacancy and silicon vacancy show notable differences in their relative accuracy when they are compared with the experimental data. This can be explained by the difference in the absolute ZFS values and the absolute errors of the calculated ZFS values that are in the same order of magnitude for the two defects. Due to the dipolar interaction, all pairs of unpaired electron spin interact and give rise to a contribution to the total ZFS tensor. In the divacancy, there is only one unpaired electron spin pair which gives a ZFS contribution in $\mathrm{GHz}$ range. However, in the silicon vacancy, there are three pairs of unpaired electron spins. Due to the high symmetry of silicon vacancy $\left(C_{3 v}\right)$, the ZFS contributions cancel out to a large extent. Note that if the silicon vacancy would have $T_{d}$ symmetry, the ZFS would be zero. On the other hand, such cancellation does not apply to the errors, thus explaining the larger relative errors for silicon vacancy.

To conclude, in this paper we have provided an identification of two common point defects in $6 \mathrm{H}-\mathrm{SiC}$ : the negatively charged silicon vacancy and neutral divacancy. Our identification was done by comparing ZPL, ZFS and hyperfine data from both theoretical calculations and experiments. The 3 different configurations for the negatively charged silicon vacancy are $k 1, k 2$, and $h$ which have the following experimental ZPL 1.368, 1.398, and $1.434 \mathrm{eV}$ respectively. For the six divacancy configurations, we identify the three $C_{3 v}$ symmetry configuration $k 1 k 1, h h$, and $k 2 k 2$ with experimental ZPL $1.088,1.092$, and $1.134 \mathrm{eV}$. Finally, for the three configurations with $C_{1 h}$ symmetry $k 1 h, k 2 k 1$, and $h k 2$ we obtain $1.103,1.119$, and $1.134 \mathrm{eV}$. Our results explains the defect configurations seen in recent experiments and may aid future 
work on defect engineering in $\mathrm{SiC}$ for quantum technologies.

No experimental hyperfine data is available yet for one of the silicon vacancy configurations and for the $C_{1 h}$ symmetry configurations in the divacancy. Once experimental data is available, this data can be compared with the theoretically calculated hyperfine tensors presented in the supplementary material and be used to further support the identification presented in this paper.

\section{SUPPLEMENTARY MATERIAL}

In the supplementary material, additional hyperfine splitting tensors are presented for silicon vacancy and divacancy.

\section{ACKNOWLEDGMENTS}

Support from the Swedish e-Science Centre (SeRC) and the Swedish Government Strategic Research Areas in Materials Science on Functional Materials at Linköping University (Faculty Grant SFO-Mat-LiU No. 2009-00971) is acknowledged. We are grateful to the support provided by Swedish Research Council (VR) Grants Nos. 2016-04068 and 2016-04810, Centre in Nano Science and Nanotechnology (CeNano), the Carl Trygger Stiftelse för Vetenskaplig Forskning (CTS 15:339), and JSPS KAKENHI A 17H01056, and 18H03770. Analysis of theoretical data was supported by the Ministry of Science and High Education of the Russian Federation in the framework of Increase Competitiveness Program of NUST MISIS (No. K2-2019-001) implemented by a governmental decree dated 16 March 2013, No 211. V.I. acknowledges the support from the MTA Premium Postdoctoral Research Program. A.G. acknowledges the support from the National Research Development and Innovation Office of Hungary (NKFIH) within the Quantum Technology National Excellence Program (Project No. 2017-1.2.1NKP-2017-00001), NVKP Program (Project No. NVKP 161-2016-0043) and Excellent Researcher Program (Grant No. KKP129886), and EU QuantERA Nanospin (NKFIH Grant No. 127902). The computations were performed on resources provided by the Swedish National Infrastructure for Computing (SNIC).

${ }^{1}$ F. Jelezko and J. Wrachtrup, physica status solidi (a) 203, 3207 (2006).

${ }^{2}$ R. Hanson and D. D. Awschalom, Nature (London) 453, 1043 (2008).

${ }^{3}$ D. D. Awschalom, L. C. Bassett, A. S. Dzurak, E. L. Hu, and J. R. Petta, Science 339, 1174 (2013).

${ }^{4}$ L. Childress, J. M. Taylor, A. S. Sørensen, and M. D. Lukin, Phys. Rev. Lett. 96, 070504 (2006).

${ }^{5}$ I. Aharonovich, S. Castelletto, D. A. Simpson, A. Stacey, J. McCallum, A. D. Greentree, and P. S., Nano Letters 9, 3191 (2009).

${ }^{6}$ R. Kolesov, K. Xia, R. Reuter, R. Stöhr, A. Zappe, J. Meijer, P. R. Hemmer, and J. Wrachtrup, Nature Communications 3, 1029 (2012).

${ }^{7}$ S. Castelletto, B. C. Johnson, V. Ivády, N. Stavrias, T. Umeda, A. Gali, and T. Ohshima, Nat. Mater. 13, 151 (2014).

${ }^{8}$ Nat Photon 10, 631 (2016).

${ }^{9}$ J. R. Weber, W. F. Koehl, J. B. Varley, A. Janotti, B. B. Buckley, C. G. Van de Walle, and D. D. Awschalom, PNAS 107, 8513 (2010).

${ }^{10} \mathrm{~J}$. Wrachtrup and F. Jelezko, Journal of Physics-Condensed Matter 18, S807 (2006).

${ }^{11}$ G. Kucsko, P. C. Maurer, N. Y. Yao, M. Kubo, H. J. Noh, P. K. Lo, H. Park, and M. D. Lukin, Nature 500, 54 EP (2013).
${ }^{12}$ A. L. Falk, P. V. Klimov, B. B. Buckley, V. Ivády, I. A. Abrikosov, G. Calusine, W. F. Koehl, A. Gali, and D. D. Awschalom, Phys. Rev. Lett. 112, 187601 (2014).

${ }^{13}$ G. Balasubramanian, I. Y. Chan, R. Kolesov, M. Al-Hmoud, J. Tisler, C. Shin, C. Kim, A. Wojcik, P. R. Hemmer, A. Krueger, T. Hanke, A. Leitenstorfer, R. Bratschitsch, F. Jelezko, and J. Wrachtrup, Nature 455, 648 (2008).

${ }^{14}$ M. W. Doherty, N. B. Manson, P. Delaney, F. Jelezko, J. Wrachtrup, and L. C. Hollenberg, Physics Reports 528, 1 (2013), the nitrogen-vacancy colour centre in diamond.

${ }^{15}$ W. F. Koehl, B. B. Buckley, F. J. Heremans, G. Calusine, and D. D. Awschalom, Nature 479, 84 (2011).

${ }^{16}$ A. L. Falk, B. B. Buckley, G. Calusine, W. F. Koehl, V. V. Dobrovitski, A. Politi, C. A. Zorman, P. X.-L. Feng, and D. D. Awschalom, Nature Commun. 4, 1819 (2013).

${ }^{17}$ V. A. Soltamov, A. A. Soltamova, P. G. Baranov, and I. I. Proskuryakov, Phys. Rev. Lett. 108, 226402 (2012).

${ }^{18}$ M. Widmann, S.-Y. Lee, T. Rendler, N. T. Son, H. Fedder, S. Paik, L.-P. Yang, N. Zhao, S. Yang, I. Booker, A. Denisenko, M. Jamali, S. A. Momenzadeh, I. Gerhardt, T. Ohshima, A. Gali, E. Janzén, and J. Wrachtrup, Nat Mater 14, 164 (2015).

${ }^{19}$ G. Balasubramanian, P. Neumann, D. Twitchen, M. Markham, R. Kolesov, N. Mizuochi, J. Isoya, J. Achard, J. Beck, J. Tissler, V. Jacques, P. R. Hemmer, F. Jelezko, and J. Wrachtrup, Nature Mater. 8, 383 (2009).

${ }^{20}$ D. J. Christle, A. L. Falk, P. Andrich, P. V. Klimov, J. U. Hassan, N. T. Son, E. Janzén, T. Ohshima, and D. D. Awschalom, Nat Mater 14, 160 (2015).

${ }^{21}$ J. Davidsson, V. Ivdy, R. Armiento, N. T. Son, A. Gali, and I. A. Abrikosov, New Journal of Physics 20, 023035 (2018).

${ }^{22}$ V. Ivády, J. Davidsson, N. T. Son, T. Ohshima, I. A. Abrikosov, and A. Gali, Physical Review B 96, 161114 (2017).

${ }^{23}$ L. Gordon, A. Janotti, and C. G. Van de Walle, Phys. Rev. B 92, 045208 (2015).

${ }^{24}$ E. Sörman, N. T. Son, W. Chen, O. Kordina, C. Hallin, and E. Janzén, Physical Review B 61, 2613 (2000).

${ }^{25}$ N. Mizuochi, S. Yamasaki, H. Takizawa, N. Morishita, T. Ohshima, H. Itoh, and J. Isoya, Physical review B 68, 165206 (2003).

${ }^{26}$ P. G. Baranov, I. Ilin, E. Mokhov, M. Muzafarova, S. B. Orlinskii, and J. Schmidt, Journal of Experimental and Theoretical Physics Letters 82, 441 (2005).

${ }^{27}$ N. T. Son, P. Carlsson, J. ul Hassan, E. Janzén, T. Umeda, J. Isoya, A. Gali, M. Bockstedte, N. Morishita, T. Ohshima, and H. Itoh, Phys. Rev. Lett. 96, 055501 (2006).

${ }^{28}$ Ö. Soykal, P. Dev, and S. E. Economou, Physical Review B 93, 081207 (2016).

${ }^{29}$ P. Hohenberg and W. Kohn, Phys. Rev. 136, B864 (1964).

${ }^{30}$ W. Kohn and L. J. Sham, Phys. Rev. 140, A1133 (1965).

${ }^{31}$ V. Ivády, I. A. Abrikosov, and A. Gali, npj Computational Materials 4, 76 (2018).

${ }^{32}$ G. Kresse and J. Hafner, Phys. Rev. B 49, 14251 (1994).

${ }^{33}$ G. Kresse and J. Furthmüller, Phys. Rev. B 54, 11169 (1996).

${ }^{34}$ P. E. Blöchl, Phys. Rev. B 50, 17953 (1994).

${ }^{35}$ G. Kresse and D. Joubert, Phys. Rev. B 59, 1758 (1999).

${ }^{36}$ J. P. Perdew, K. Burke, and M. Ernzerhof, Phys. Rev. Lett. 77, 3865 (1996).

${ }^{37}$ J. Heyd, G. E. Scuseria, and M. Ernzerhof, J. Chem. Phys. 118, 8207 (2003).

${ }^{38}$ J. Heyd, G. E. Scuseria, and M. Ernzerhof, J. Chem. Phys. 124, 219906 (2006).

${ }^{39}$ A. Gali, E. Janzén, P. Deák, G. Kresse, and E. Kaxiras, Phys. Rev. Lett. 103, 186404 (2009).

${ }^{40}$ V. Ivády, T. Simon, J. R. Maze, I. A. Abrikosov, and A. Gali, Phys. Rev. B 90, 235205 (2014).

${ }^{41}$ K. Szász, T. Hornos, M. Marsman, and A. Gali, Phys. Rev. B 88, 075202 (2013).

${ }^{42}$ A. L. Falk, P. V. Klimov, V. Ivády, K. Szász, D. J. Christle, W. F. Koehl, A. Gali, and D. D. Awschalom, Phys. Rev. Lett. 114, 247603 (2015).

${ }^{43}$ E. Janzén and B. Magnusson, in Silicon Carbide and Related Materials 2004, Materials Science Forum, Vol. 483 (Trans Tech Publications, 2005) pp. 341-346.

${ }^{44}$ T. Biktagirov, W. G. Schmidt, U. Gerstmann, B. Yavkin, S. Orlinskii, P. Baranov, V. Dyakonov, and V. Soltamov, Physical Review B 98, 195204 (2018). 